\title{
Metformin versus Insulin for Gestational Diabetes: A Randomized Clinical Trial
}

\author{
Ajay Agrawal ${ }^{1}$, Shailaja Chhetri ${ }^{1}$, Jyoti Agrawal $^{2}$, Robin Maskey ${ }^{3}$ \\ ${ }^{1}$ Department of Obstetrics and Gynaecology, BPKIHS, ${ }^{2}$ Department of Paediatrics and Adolescent \\ Medicine, BPKIHS, ${ }^{3}$ Department of Internal Medicine, BPKIHS
}

\begin{abstract}
Background: Insulin therapy is often started if medical nutritional therapy (MNT) fails to manage Gestational diabetes mellitus (GDM) which is associated with multiple injections and demands more patient compliance. So use of safe and effective oral agents may offer advantages over insulin. Objectives: To evaluate glycaemic control in women receiving metformin versus insulin for GDM, and to identify factors predicting the need for supplemental insulin in women initially treated with metformin. Methods: Women, $18-45$ years at 20-33 weeks of gestation with singleton pregnancy with GDM without satisfactory glycemic control on MNT for a minimum period of 1 week were randomised to receive either insulin or metformin. Results: There was no significant difference in mean pre-treatment glucose levels between two groups $(\mathrm{P}=0.890)$. After randomizing, women received their respective intervention. Mean glucose level measured after glycaemic control showed, lower levels in the metformin group $(\mathrm{P}=.034)$. Also women under metformin presented less weight gain $(\mathrm{P}=.02)$ and a lower frequency of neonatal hypoglycaemia $(\mathrm{P}=.032)$. Thirteen women in the metformin group (31.7\%) required supplemental insulin. Early gestational age at diagnosis and high BMI were identified as predictors of the need for supplemental insulin. Conclusions: Metformin appears to constitute safe and effective treatment option for GDM who do not have satisfactory glycemic control. It was found to provide adequate glycemic control with lower mean glucose level, less weight gain and a lower frequency of neonatal hypoglycaemia. Early gestational age at diagnosis and high BMI were predictors of the need for supplemental insulin therapy in women initially treated with metformin.
\end{abstract}

Key Words: Gestational Diabetes, Insulin, Metformin

\section{INTRODUCTION}

Gestational diabetes mellitus (GDM), affecting $5 \%$ of population, has classically been defined as any glucose intolerance first identified during pregnancy ${ }^{1}$. American Diabetes Association (ADA) defined it as "Diabetes diagnosed in the second or third trimester of pregnancy that is not clearly overt diabetes"2. As per IADPSG criteria, women can be diagnosed to have GDM even in the first trimester, if fasting plasma glucose (FPG) is $\geq 5.1 \mathrm{mmol} / \mathrm{L}$ $(92 \mathrm{mg} / \mathrm{dL})$, but $\leq 7 \mathrm{mmol} / \mathrm{L}(126 \mathrm{mg} / \mathrm{dL})^{3}$.

Studies indicate that the severity of maternal and

\section{Correspondence Author}

Dr Ajay Agrawal, Additional Professor,1 Department of OBGYN, BPKIHS, Email- drajayagrawal1980@gmail.com, Phone- 9852049451 fetal complications is proportional to the level of maternal hyperglycemia ${ }^{4-6}$. The benefits of treating GDM with diet and insulin, if necessary, are well established ${ }^{7.8 .}$ However women who begin insulin require education to ensure the safe administration of insulin. So use of safe and effective oral agents may offer advantages over insulin because of their ease of use and lower cost.

Investigations on the use of metformin for the treatment of GDM have concluded that metformin seems to be an effective alternative for the treatment of $\mathrm{GDM}^{9-12}$. However, response to treatment in patients with gestational diabetes is highly dependent on patient characteristics. ${ }^{13}$ Since Nepal is inhabited by mixture of different cast and 
ethnicity which is different from population in other part of the world, we need to test the response of metformin in GDM in our population. Thus this present study is conducted with primary aim to compare glycemic control in women who received metformin versus standard use of insulin for the treatment of GDM in our population. Our secondary objective was to compare neonatal outcome among women in two groups and identify factors that lead to need for insulin in women under metformin.

\section{METHODS}

This was a randomised controlled study done over two years from May 2016 to January 2018, involving women with diagnosed GDM not controlled with MNT for a minimum period of 1 week at BP Koirala Institute of Health sciences (BPKIHS), Dharan, Nepal. Women, 18-45 years, who were at 20-33 weeks of gestation having singleton pregnancy, were included. Women with contraindication to taking metformin, pre-pregnancy diagnosis of diabetes, any obstetrical indication for immediate vaginal or surgical delivery and having fetal congenital malformation were excluded. Total of 82 Women who met selection criteria were included in this study. Consent was taken from women before enrolling them to this study. This study was approved by Institutional Review Committee, BPKIHS (IRC/480/015).

After selection women were randomised using computer generated random number table into 2 groups, 41 in each. Women in Group 1, taken as cases, were started with Tab Metformin, 1500mg in 3 divided doses taken with food and increased to maximum of $2500 \mathrm{mg}$ depending upon glycemic control till the target blood sugar was met. Metformin was stopped if significant maternal conditions, such as severe preeclampsia, sepsis, or pregnancy cholestasis and also if fetal growth restriction developed. Women in Group 2, as control, received standard Insulin therapy as per our hospital protocol. They were typically started with combination of regular and intermediate acting insulin according to their weight and were adjusted to meet the target blood sugar. The target glucose reference values recommended by the ADA were used: fasting $(95 \mathrm{mg} / \mathrm{dL})$ and 2 hours after a meal $(120 \mathrm{mg} / \mathrm{dL})^{14}$. Women in group 1 who didn't tolerate metformin or who didn't achieve target glucose level were supplemented with insulin.

At study entry, background maternal demographic data, medical history, family history, obstetric history, medication intake through pregnancy, early pregnancy data, and any pregnancy complications were recorded. Paternal demographic data and height and weight were also recorded. Fetal ultrasound growth within 2 weeks before or 1 week after study entry was documented. During the study, women were asked to continue measuring capillary glucose levels fasting and 2 hour after the start of each meal regularly weekly, self by glucometer as per instructions and report to the investigator. At delivery, pregnancy complications, indication for induction (if performed), mode of delivery, and complications are recorded from the hospital notes. Detailed neonatal morbidity is also recorded. Trained personnel performed anthropometric and blood sugar measurements on the baby within 48 $\mathrm{h}$ of birth.

Numerical variables were compared by the Student $\mathrm{t}$ test or Mann-Whitney test. The $x 2$ test, Fisher exact test or likelihood ratio tests were used to compare categorical variables. In addition, logistic regression analysis was performed to predict the need for supplemental insulin in women initially treated with metformin.

\section{RESULTS}

In this study 82 women were enrolled and they were randomised into two groups with 41 in each group. The demographic and clinical characters in two groups were recorded at enrolment. This shows similar pattern as shown in Table-1. It includes age, body mass index (BMI) at enrolment, gestational week and parity. We also recorded fasting blood sugar after overnight fasting and post prandial as well as mean pre-treatment blood glucose level and glycated haemoglobin at enrolment. Also blood test was done for liver function as well as renal function at enrolment to make sure this result does 
Metformin versus Insulin for Gestational Diabetes: A .......

Jour of Diab and Endo Assoc of Nepal 2019; 3 (2): (3-8)

ISSN Print 2594-3367 ISSN Online 2631-2107

not preclude the use of metformin. There was no significant difference in mean pre-treatment glucose levels between two groups $(\mathrm{P}=0.890)$. Also the glycated haemoglobin was similar in both the group. After enrolment in the study, patients were randomised into two groups as described in methods. After randomizing, women received their respective intervention.

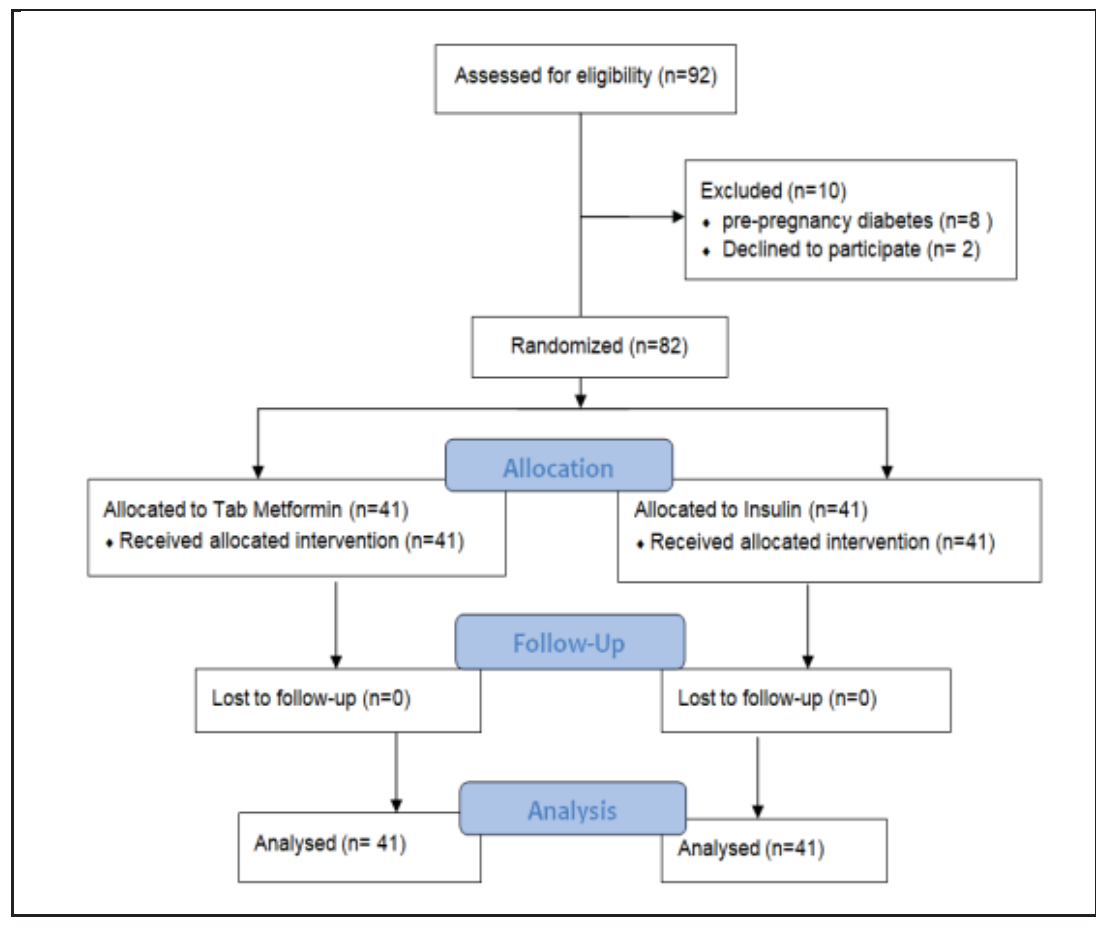

Figure1. Enrollment of Subjects.

Table.1 Baseline maternal Characterstics

\begin{tabular}{|l|l|l|}
\hline Character & Metformin (41) & Insulin (41) \\
\hline Age (yrs) & $33.4 \pm 5.4$ & $33.0 \pm 5.3$ \\
\hline BMI at enrolment & $35.1 \pm 7.2$ & $34.6 \pm 8.3$ \\
\hline Period of gestation (weeks) & $30.3 \pm 3.2$ & $31.2 \pm 3.1$ \\
\hline Nulliparous(\%) & 31.7 & 31.9 \\
\hline Glycated haemoglobin & $5.7 \pm 0.2$ & $5.8 \pm 0.8$ \\
\hline
\end{tabular}

Less weight gain was observed in women in group 1 compared to group 2 between the start of medication treatment and delivery (group 1: $0.53 \pm 2.52 \mathrm{~kg}$ vsgroup $2: 2.3 \pm 2.77 \mathrm{~kg} ; \mathrm{P}=.002$ ). There was no difference in the two groups in terms of frequency of preeclampsia, prematurity and operative delivery.

None of the women discontinued the study protocol (figure 1). Only 10 women in metformin group reported some side effects, most frequent being gastrointestinal effects like nausea and occasional increase frequency of bowel movements. But all of them continued with the treatment protocol. Out of 41 women in group 1, $13(31.7 \%)$ required supplemental insulin to achieve target glycemic control. Regarding glucose control, the mean glucose level measured after glycemic control showed, lower levels in the metformin group $(\mathrm{P}=$ .034 ) compared to insulin group. (Table 3 ) 
Table 3 Mean blood glucose level after treatment.

\begin{tabular}{|l|l|l|l|}
\hline Pretreatment blood glucose & Fasting & 2 Hr- post prandial & P value \\
\hline Metformin & $102.15 \pm 21.96$ & $120.61 \pm 22.63$ & \multirow{2}{*}{0.890} \\
\cline { 1 - 3 } Insulin & $100.87 \pm 15.05$ & $123.72 \pm 19.4$ & \\
\hline Post-treatment blood glucose & & & \\
\cline { 1 - 3 } Metformin & $90.09 \pm 16.29$ & $106.87 \pm 11.16$ & \multirow{2}{*}{0.034} \\
\cline { 1 - 3 } Insulin & $88.35 \pm 7.45$ & $111.43 \pm 8.84$ & \\
\hline
\end{tabular}

\section{Neonatal outcome}

No significant differences between the 2 groups were observed regarding thefollowingimmediate neonatal outcomes: gestational age at birth (group 1: $38.33 \pm 1.45$ weeks vs group 2: $38.24 \pm 1.53$ weeks; $\mathrm{P}=0.776$ ), 1-minute Apgar score (group 1: 9 [0-10] vs group 2: 9 [4-10]; $\mathrm{P}=.980$ ), 5-minute Apgar score (group 1: $10[0-10]$ vs group 2: 10 [0-10]; $\mathrm{P}=.188$ ) and newborn weight (group 1: $3143.7 \pm 446.6 \mathrm{~g}$ vs group 2: $3237.6 \pm 586.8 \mathrm{~g} ; \mathrm{P}=.390$ ) (Table 2). There were no fetuses with macrosomia in the group metformin vs 3 $(7.3 \%)$ cases in the insulin group $(\mathrm{P}=.342)$. A lower frequency of neonatal hypoglycaemia was observed in cases treated with metformin $(3 / 41,7.3 \%)$ compared with newborns from the insulin group $(10 / 41,24.3 \%)$ $(\mathrm{P}=.042)$.

Table 2 - Neonatal outcome

\begin{tabular}{|l|l|l|l|}
\hline Variables & Metformin & Insulin & P value \\
\hline Gestational age at birth (weeks) & $38.33 \pm 1.45$ & $38.24 \pm 1.53$ & 0.776 \\
\hline 1-minute Apgar score & $9[0-10]$ & $9[4-10]$ & 0.980 \\
\hline 5-minute Apgar score & $10(0-10)$ & $10(0-10)$ & 0.188 \\
\hline Newborn weight & $3143.7 \pm 446.6 \mathrm{~g}$ & $3237.6 \pm 586.8 \mathrm{~g}$ & 0.390 \\
\hline
\end{tabular}

Early gestational age at diagnosis (odds ratio.0.78; $95 \%$ confidence interval, $0.52-0.97 ; \mathrm{P}=.02$ ) and high BMI were identified as predictors of the need for insulin by logistic regression analysis.

\section{DISCUSSSION}

As per the primary objective of this study we were able to evaluate glycemic control in both the groups of women. The mean glucose level measuredafterglycemic control showed, lower levels in the metformin group $(\mathrm{P}=.034)$ compared to insulin group (Table 3). Similarresults were shown in the study by Spaulonci CP et al. They also demonstrated that lower level of blood sugar was observed especiallyafter dinner ${ }^{12}$. Less weight gain observed in women of group 1 compared to group 2 between the start of medication treatment and delivery (group 1: $0.53 \pm 2.52 \mathrm{~kg}$ vsgroup 2: $2.3 \pm 2.77 \mathrm{~kg} ; \mathrm{P}=.002$ ) in our study was again comparable to other similar study ${ }^{10,12}$. Also as comparable to Spaulonci CP et a $1^{12}$ and Rowan et al 10 , there was no difference in the two groups in terms of frequency of preeclampsia, prematurity and operative delivery.

In the present study, only ${ }^{10}(24.3 \%)$ women in metformin group reported some side effects, but all of them continued with the treatment protocol. Twenty-one (45.65\%) of the 46 womenwho received metformin reported someside effect in the study by Spaulonci CP et al ${ }^{12}$ which is similar to our study. Out of 41 women in group ${ }^{1,13}(31.7 \%)$ required supplemental insulin to achieve target glycemic control. This is more that that reported by Spaulonci $\mathrm{CP}$ et al ${ }^{12}$ who reported ${ }^{12}(26.08 \%)$ women in metformin group requiring supplemental insulin. In the study by Rowan et al ${ }^{10} 46.3 \%$ of women taking metforminrequired supplemental insulin. These 
differencesmay be because of difference in ethnicity and characteristic of population as diabetes widely varies among different population.

Regarding immediate neonatal outcomes like gestational age at birth, 1,5-minute Apgar score and newborn weight, our study showed no significant differences between the 2 groups. There were no fetus with macrosomia in the group metformin vs $3(7.3 \%)$ cases in the insulin group $(\mathrm{P}=.342)$. In the study by Rowan et al ${ }^{10}$ the primary outcome, a composite of neonatal hypoglycemia, respiratory dis $\neg$ tress, need for phototherapy, birth trauma, 5-minute Apgar score lower than ${ }^{7}$, or pre $\neg$ maturity, occurred with similar frequency in the 2 groups (32\% in each group) where 733 women were randomised to metformin versus insulin. Our study also demonstrated lower frequency of neonatal hypoglycaemia in cases treated with metformin (3/41, 7.3\%) compared with newborns from the metformin group $(10 / 41,24.3 \%)(\mathrm{P}=.042)$ which was comparable to other studies ${ }^{10,12}$. As per the literature review our women with early gestational age at diagnosis and high BMI were identified as predictors of the need for insulin.

The strength of this study is that all women were followed up till delivery. Our group of women included all different caste of Nepal so the results can be implemented to all. Major limitations are we don't have any records of level of glycemic control at home because of poor patient compliance and Cord blood has not been stored for assessment of insulin and c peptide.

\section{CONCLUSION}

The primary objective of this study was to evaluate glycemic control in women with GDM treated with metformin or insulin.Metformin appears to constitute safe and effective treatment option for GDM who do not have satisfactory glycemic control with MNT. It was found to provide adequate glycemic control with lower mean glucose level, less weight gain and a lower frequency of neonatal hypoglycemia. Early gestational age at diagnosis and high BMI were predictors of the need for supplemental insulin therapy in women initially treated with metformin

\section{References}

1. American Diabetic Association. Diagnosis and classification of diabetes mellitus. Diabetes Care. 2006;29(Suppl 1):S43-S48.

2. American Diabetic Association. Classification and diagnosis of diabetes mellitus. Diabetes Care. 2015;38(Suppl 1):S8-S16.

3. International Association of Diabetes and Pregnancy Study Groups Consensus Panel, Metzger BE, Gabbe SG, Persson $\mathrm{B}$, et al. International association of diabetes and pregnancy study groups recommendations on the diagnosis and classification of hyperglycemia in pregnancy. Diabetes Care. 2010;33(3):676-682.

4.Miller E, Hare JW, Cloherty JP, Dunn PJ, Gleason RE, Soeldner JS, et al. Elevated maternalhemoglobin A1c in early pregnancy and major congenital anomalies in infants of diabetic mothers. N Engl J Med. 1981 May 28;304(22):1331-4.

5. Lucas MJ, Leveno KJ, Williams ML, Raskin P, Whalley PJ. Early pregnancy glycosylatedhemoglobin, severity of diabetes, and fetal malformations. Am J Obstet Gynecol. 1989Aug;161(2):426-31.

6.Temple R, Aldridge V, Greenwood R, Heyburn P, Sampson M, Stanley K. Associationbetween outcome of pregnancy and glycaemic control in early pregnancy in type 1diabetes: population based study. BMJ. 2002 Nov 30;325(7375):1275-6.

7.Crowther CA, Hiller JE, Moss JR, McPhee AJ, Jeffries WS, Robinson JS; Australian Carbohydrate Intoler $\neg$ ance Study in Pregnant Women (ACHOIS) Trial Group. Effect of treatment of gestational diabetes mellitus on pregnancy outcomes. N Engl J Med. 2005;352(24):2477-2486.

8. Landon MB, Spong CY, Thom E, et al; Eunice Ken $\neg$ nedy Shriver National Institute of Child Health and Human Development Maternal-Fetal Medicine Units Network. A multicenter, randomized trial of treat $\neg$ ment for mild gestational diabetes. N Engl J Med. 2009;361(14):1339-1348.

9. Moore LE, Briery CM, Clokey D, et al. Metforminand insulin in the management of gestationaldiabetes mellitus: preliminary results of acomparison. J Reprod Med 2007;52:1011-5.

10. Rowan JA, Hague WM, Gao W, BattinMR,Moore MP. Metformin versus insulin for thetreatment of gestational diabetes. N Engl J Med2008;358:2003-15. 
11. Nicholson W, Bolen S, Witkop CT, Neale D,Wilson L, Bass E. Benefits and risks of oral diabetesagents compared with insulin in womenwith gestational diabetes: a systematic review.ObstetGynecol 2009;113:193-205.

12. Spaulonci CP, Bernardes LS, Trindade TC, et al. Randomized trial of metformin vs insulin in the management of gestational diabetes. Am J Obstet

Gynecol 2013;209:34.e1-7.
Journal of Diabetes and Endocrinology

Association of Nepal
13. Sapienza AD, Francisco RP, Trindade TC, Zugaib M. Factors predicting the need for insulin therapy in patients with gestational diabetes mellitus. Diabetes Res ClinPract 2010;88:81-6.

14. Metzger BE, Buchanan TA, Coustan DR, et al. Summary and recommendations of the Fifth International Workshop-Conference on Gestational Diabetes Mellitus. Diabetes Care 2007;30(Suppl 2):S251-60. 